\title{
Analytical Performance Modelling of Slotted Surface-Mounted Permanent Magnet Machines with Rotor Eccentricity
}

\author{
Bo Yan*, Xiuhe Wang ${ }^{\dagger}$ and Yubo Yang*
}

\begin{abstract}
This paper presents an improved subdomain method to predict the magnet field distributions and electromagnetic performance of the surface-mounted permanent magnet (SPM) machines with static or dynamic eccentricity. Conventional subdomain models are either based on the scalar magnet potential to predict the rotor eccentricity effect or dependent on the magnetic vector potential without considering the eccentric rotor. In this paper, both the magnetic vector potential and the perturbation theory are introduced in order to accurately calculate the effect of rotor eccentricity on the open-circuit and armature reaction performance. The calculation results are presented and validated by the corresponding finite-element method (FEM) results.
\end{abstract}

Keywords: FEM, Magnetic vector potential, Rotor eccentricity, SPM machine, Subdomain method

\section{Introduction}

Due to the favourable characteristics such as the high efficiency, high power density and easy maintenance, the SPM machines are widely used in many applications, ranging from industrial servos and domestic appliances to medical facilities. However, as one common phenomenon caused by the unavoidable manufacture tolerance in mass production, the rotor eccentricity can greatly affect the magnetic field distribution and bring about problems related with mechanical vibration and acoustic noise.

Two basic approaches, including the FEM and the analytical method, are commonly carried out to investigate the effect of rotor eccentricity on the performance of SPM machines. As one numerical method capable to take the effect of magnetic saturation, flux leakage and complex structure into consideration, the FEM is widely utilized in the performance analysis of electric machines. The FEM is adopted in [1] to calculate the air gap flux density of SPM and interior permanent magnet (IPM) machine with rotor eccentricity. The unbalanced magnetic pull (UMP) of brushless PM machine with rotor eccentricity is calculated by FEM in [2], and it is found that the UMP is mostly loadindependent for the machine with strong and thick PMs. The electromagnetic force of brushless PM machine with rotor eccentricity is studied with FEM in [3], and special rotor configuration is designed to reduce the harmonic amplitude. In [4], the effect of rotor eccentricity on the cogging torque of the PM machine is investigated according to the FEM prediction. The 3D transient FEM is applied in [5] to investigate the effects of dynamic rotor eccentricity in the single-rotor single-stator axial flux

$\dagger$ Corresponding Author: School of Electrical Engineering, Shandong Univerity, Jinan, China. (sdpmsm@gmail.com)

* School of Electrical Engineering, Shandong Univerity, Jinan, China

(jabourne123@163.com, ybyang@sdu.edu.cn)

Received: June 30, 2016 ; Accepted: December 12, 2016 permanent magnet (AFPM) synchronous machine and different eccentricity conditions are considered.

Compared to FEM, the analytical method is time saving and can give enough insight in the initial design and optimization of electric machines. In [6] and [7], the magnetic equivalent circuit (MEC) is adopted to study the effect of rotor eccentricity on the performance of PM machines, and the effect of magnetic saturation is also considered. In [8-11], the conformal transformation is adopted to describe the effect of rotor eccentricity on the performance of PM machines. In [12], the cogging torque of SPM machine with rotor eccentricity is predicted by combination of the FEM and analytical methods. It is found that the eccentricity effects are closely related to the combination of poles and slots number. In [13], analytical method based on the scalar magnetic potential is introduced to calculate the unbalanced magnetic forces in the toroidally wound brushless dc (BLDC) motor with rotor eccentricity, and the analytical model is verified by the FEM results.

The subdomain method obtains the magnetic potential expressions in all subregions by solving the governing equations, and has better accuracy than the above analytical methods applied in [6-13]. In [14] and [15], the subdomain model is firstly introduced to analyse the effect of rotor eccentricity on the air gap flux density of SPM machine, and the perturbation method is adopted to consider the effect of rotor eccentricity. In [16], the subdomain model of open-slot SPM machine with rotor eccentricity is developed, and the effect on the flux density is studied. In [17], the subdomain model of SPM machine with rotor eccentricity and PM imperfections is advised, and the effect on the cogging torque is studied. In [18], the subdomain model of slotless SPM machine with rotor eccentricity and different magnetization patterns is advised, and the effect on the no-load performance is studied. 
The above papers [14-18] based on subdomain method have provided comprehensive discussions and analysis about the SPM machines with rotor eccentricity. However, due to the application limitation of the scalar magnetic potential, only the no-load performance can be considered, and with no regard to the effect of slots or the tooth tips. The models in these papers can only be applied to the slotless or open-slot SPM machines.

Compared to the above-mentioned methods, an accurate subdomain model consisting of the stator slots and toothtips is presented in this paper, and based on the magnetic vector potential, the rotor eccentricity effect on both noload operation and armature reaction can be fully discussed and calculated. Furthermore, the effects of various combinations of pole-number and slot-number on the performance of SPM machine with rotor eccentricity are investigated. It is also found that the effects of rotor eccentricity on the flux linkage distributions vary greatly among the SPM machines with different winding arrangements.

\section{Subdomain Model with Rotor Eccentricity}

The following assumptions are made for the analytical modelling:

1) the permeability of iron core is set as infinity.

2) the end effect and eddy currents are neglected.

3) the PMs have linear demagnetization characteristics.

4) the relative permeability of air between magnets is the same as the adjacent magnets.

\subsection{Rotor eccentricity and coordinate systems trans- form}

As shown schematically in Fig. 1(a) and (b), two SPM machines with rotor eccentricity, including the 8-pole/ 12 slot $(8 \mathrm{p}-12 \mathrm{~s})$ one and the 8 -pole/9-slot $(8 \mathrm{p}-9 \mathrm{~s})$ one, are given for analysis. The air gap length is amplified for demonstration clarity. The whole field is divided into four sub-regions: the PMs, the air-gap, the slot openings and the slots. They are labelled as region $1,2,3 i$ and $4 i$ in insideout sequence, where $i$ indicates the $i$ th stator slot opening or slot.

As shown is Fig. 1c, when accounting for the rotor eccentricity, which means the stator and rotor centers don't coincide with each other, two coordinate systems are necessarily adopted [14]: the stator or $r-\theta$ coordinate and the rotor or $\rho-\psi$ coordinate are respectively attached to the stator center $O_{s}$ and the rotor center $O_{r}$. The $r-\theta$ coordinate system remains stationary in space, while the $\rho-\psi$ coordinate system whirls around axis $O_{r}$ with rotation velocity $\omega_{r}$ and initial angular position $\theta_{0}$. In the $r-\theta$ coordinate, the position of rotor center $O_{r}$ can be expressed as $(\varepsilon, \phi)$, in which $\varepsilon$ means the offset distance of rotor center to the stator center and $\phi$ indicates the included

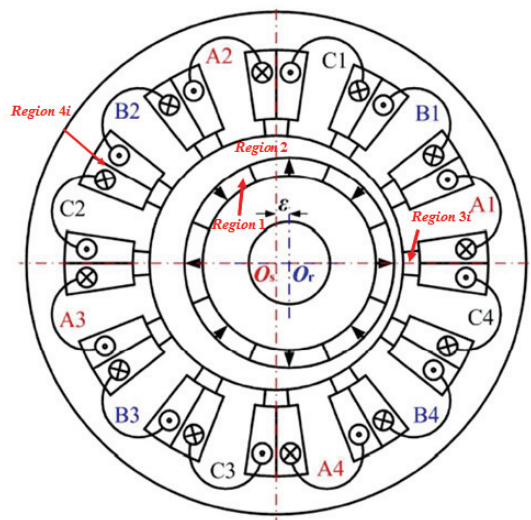

(a) The $8 p-12$ s SPM machine with rotor eccentricity

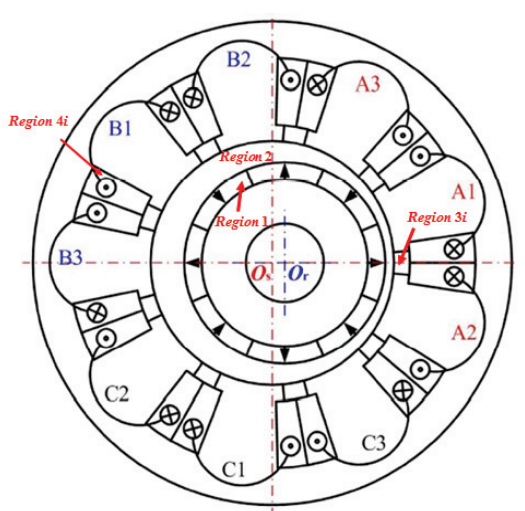

(b) The $8 \mathrm{p}-9 \mathrm{~s}$ SPM machine with rotor eccentricity

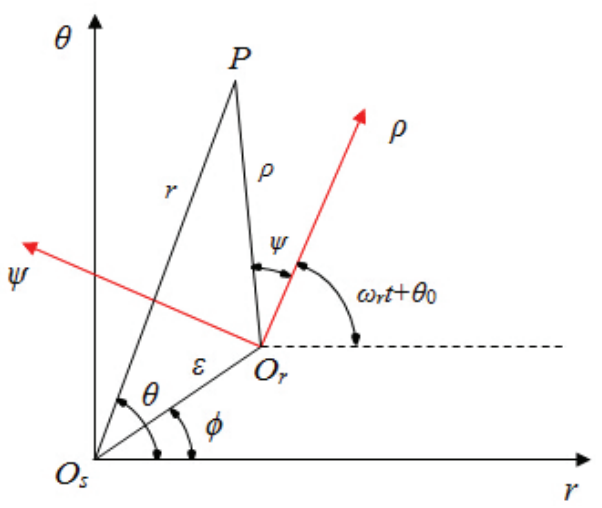

(c) The stator and rotor coordinate systems

Fig. 1. The sub-regions and coordinate systems

angle between the segment $O_{s}-O_{r}$ and $r$-axis.

The eccentricity can be classified as three types: static eccentricity, dynamic eccentricity and mixed eccentricity [14]. For the static one, both $\varepsilon$ and $\phi$ remain constant since the rotor whirls around its own center axis. For the dynamic eccentricity, as the rotation axis of the rotor converts to the stator center, $\varepsilon$ is invariant but $\phi$ changes with the time. As to the mixed eccentricity, the rotation axis is neither the rotor center nor the stator center, and both $\varepsilon$ and $\phi$ change with time.

Any point $P$ can be expressed as $(r, \theta)$ in the stator 
coordinate and $(\rho, \psi)$ in the rotor coordinate, where $r$ and $\rho$ are the polar distances and $\theta$ and $\psi$ are the polar angles. The position factors of the rotor coordinate can be expressed by the counterparts of the stator coordinate as [14]:

$$
\left\{\begin{array}{l}
\rho=r-\varepsilon \cos (\theta-\phi)+O\left(\varepsilon^{2}\right) \\
\psi=\theta-\omega t+\frac{\varepsilon}{r} \sin (\theta-\phi)+O\left(\varepsilon^{2}\right)
\end{array}\right.
$$

where $t$ is the time.

\subsection{Perturbation theory and governing equations}

Rotor eccentricity can be treated as one kind of the perturbation phenomena, which can be quantified by the following expansion form when only terms up to the first order are taken into consideration [14]:

$$
X(r, \theta, \delta)=X^{(0)}(r, \theta)+\delta \cdot X^{(1)}(r, \theta)
$$

where $X$ is the physical quantity accounting for perturbation, $\delta$ is the perturbation factor and $X^{(0)}$ and $X^{(1)}$ are the zeroth- and first-order values.

According to the expansion, the magnetic vector potential in the whole domain can be given as:

$$
A(r, \theta, \mathcal{E})=A^{(0)}(r, \theta)+\varepsilon \cdot A^{(1)}(r, \theta)
$$

where $A$ is the magnetic vector potential in the 2D field, $A^{(0)}$ and $A^{(1)}$ are components corresponding to the zeroth and first orders.

When the rotor is not eccentric, viz. $\varepsilon=0$, the expression (3) indicates the magnetic vector potential with normal rotor. Since detailed analysis of magnetic vector potential for SPM machine without rotor eccentricity have been made in [19]-[21], only the first-order component, viz. $A^{(1)}$, is discussed in this paper.

As the additional component, $A^{(1)}$ is completely generated by the rotor eccentricity effect without magnets or currents excitation. Consequently, $A^{(1)}$ in all subdomains can be governed by the Laplacian equations:

$$
\begin{aligned}
& \frac{\partial^{2} A_{z 1}^{(1)}}{\partial r^{2}}+\frac{1}{r} \frac{\partial A_{z 1}^{(1)}}{\partial r}+\frac{1}{r^{2}} \frac{\partial^{2} A_{z 1}^{(1)}}{\partial \theta^{2}}=0 \\
& \frac{\partial^{2} A_{z 2}^{(1)}}{\partial r^{2}}+\frac{1}{r} \frac{\partial A_{z 2}^{(1)}}{\partial r}+\frac{1}{r^{2}} \frac{\partial^{2} A_{z 2}^{(1)}}{\partial \theta^{2}}=0 \\
& \frac{\partial^{2} A_{z 3 i}^{(1)}}{\partial r^{2}}+\frac{1}{r} \frac{\partial A_{z 3 i}^{(1)}}{\partial r}+\frac{1}{r^{2}} \frac{\partial^{2} A_{z 3 i}^{(1)}}{\partial \theta^{2}}=0 \\
& \frac{\partial^{2} A_{z 4 i}^{(1)}}{\partial r^{2}}+\frac{1}{r} \frac{\partial A_{z 4 i}^{(1)}}{\partial r}+\frac{1}{r^{2}} \frac{\partial^{2} A_{z 4 i}^{(1)}}{\partial \theta^{2}}=0
\end{aligned}
$$

where $A_{z 1}^{(1)}, A_{z 2}^{(1)}, A_{z 3 i}^{(1)}$, and $A_{z 4 i}^{(1)}$ are the magnetic vector potentials in the PMs, the air gap, the $i$ th stator slot opening and stator slot, and general solutions to Eq. (4) - (7) can be derived as:

$$
\begin{aligned}
A_{z 1}^{(1)}=\sum_{k}\left[A_{1}^{(1)}\left(\frac{r}{R_{m}}\right)^{k}+B_{1}^{(1)}\left(\frac{r}{R_{r}}\right)^{-k}\right] \cos (k \theta) \\
+\sum_{k}\left[C_{1}^{(1)}\left(\frac{r}{R_{m}}\right)^{k}+D_{1}^{(1)}\left(\frac{r}{R_{r}}\right)^{-k}\right] \sin (k \theta) \\
A_{z 2}^{(1)}=\sum_{k}\left[A_{2}^{(1)}\left(\frac{r}{R_{s}}\right)^{k}+B_{2}^{(1)}\left(\frac{r}{R_{m}}\right)^{-k}\right] \cos (k \theta) \\
+\sum_{k}\left[C_{2}^{(1)}\left(\frac{r}{R_{s}}\right)^{k}+D_{2}^{(1)}\left(\frac{r}{R_{m}}\right)^{-k}\right] \sin (k \theta) \\
A_{z 3 i}^{(1)}=\sum_{m}\left[C_{3 i}^{(1)}\left(\frac{r}{R_{t}}\right)^{F_{m}}+D_{3 i}^{(1)}\left(\frac{r}{R_{s}}\right)^{-F_{m}}\right] \\
\times \cos \left[F_{m}\left(\theta-\theta_{i}+b_{o a} / 2\right)\right] \\
A_{z 4 i}^{(1)}=\sum_{n} D_{4 i}^{(1)}\left[\left(\frac{R_{t}}{R_{s b}}\right)^{E_{n}}\left(\frac{r}{R_{s b}}\right)^{E_{n}}+\left(\frac{r}{R_{t}}\right)^{-E_{n}}\right] \\
\times \cos \left[E_{n}\left(\theta-\theta_{i}+b_{s a} / 2\right)\right]
\end{aligned}
$$

where $A_{1}^{(1)}, B_{1}{ }^{(1)}, C_{1}^{(1)}, D_{1}^{(1)}, A_{2}^{(1)}, B_{2}^{(1)}, C_{2}^{(1)}, D_{2}^{(1)}, C_{3 i}{ }^{(1)}$, $D_{3 i}{ }^{(1)}$, and $D_{4 i}{ }^{(1)}$ are the integration constants, $\theta_{i}$ is the angular position of the center line corresponding to the $i$ th stator slot or the slot opening, $R_{r}$ and $R_{m}$ are the inner and outer radius of PMs, $R_{s}$ and $R_{t}$ are the inner and outer radius of slot opening, $R_{s b}$ is the outer radius of stator slot, $b_{o a}$ is the width angle of the slot opening, $b_{s a}$ is the width angle of the slot, and

$$
\left\{\begin{array}{l}
F_{m}=m \pi / b_{o a} \\
E_{n}=n \pi / b_{s a}
\end{array}\right.
$$

\subsection{Boundary conditions and determination solutions}

The effect of the rotor eccentricity can be found at the rotor yoke surface and the interface between the PMs and air gap in the $r-\theta$ coordinate. In the $\rho-\psi$ coordinate, the radii corresponding to the surface and interface are $R_{r}$ and $R_{m}$, respectively. By applying $\rho=R_{r}$ and $R_{m}$ to the first part in Eq. (1), the following equations can be obtained as:

$$
\begin{aligned}
& r-\varepsilon \cos (\theta-\varphi)-R_{r}+O\left(\varepsilon^{2}\right)=0 \\
& r-\varepsilon \cos (\theta-\varphi)-R_{m}+O\left(\varepsilon^{2}\right)=0
\end{aligned}
$$

Eq. (13) and (14) can be defined as the specific functions, which illuminate the relationship between the position factors $(r$ and $\theta$ ) at the boundaries in the stator coordinate. The functions can be rewritten as: 


$$
\begin{aligned}
& f_{\text {rotor }}(r, \theta, \mathcal{E}) \\
= & r-\varepsilon \cos (\theta-\varphi)-R_{r}+O\left(\varepsilon^{2}\right)=0 \\
& f_{P M \text {-air }}(r, \theta, \varepsilon) \\
= & r-\varepsilon \cos (\theta-\varphi)-R_{m}+O\left(\varepsilon^{2}\right)=0
\end{aligned}
$$

The normal vectors of these boundaries can then be derived as:

$$
\begin{aligned}
\mathbf{n}_{\text {rotor }}(r, \theta, \varepsilon) & =\nabla f_{\text {rotor }} \\
& =\mathbf{e}_{\mathbf{r}}+\frac{\varepsilon}{r} \sin (\theta-\varphi) \mathbf{e}_{\boldsymbol{\theta}}+O\left(\varepsilon^{2}\right) \\
\mathbf{n}_{P M \text {-air }}(r, \theta, \varepsilon) & =\nabla f_{P M \text {-air }} \\
& =\mathbf{e}_{\mathbf{r}}+\frac{\varepsilon}{r} \sin (\theta-\varphi) \mathbf{e}_{\boldsymbol{\theta}}+O\left(\varepsilon^{2}\right)
\end{aligned}
$$

The boundary conditions at rotor core surface and PMsairgap interface can be described by:

$$
\begin{gathered}
\mathbf{n}_{\text {rotor }} \times \mathbf{H}_{1}=\mathbf{0} \\
\mathbf{n}_{P M-\text { air }} \times\left(\mathbf{H}_{1}-\mathbf{H}_{2}\right)=\mathbf{0} \\
\mathbf{n}_{P M \text {-air }} \cdot\left(\mathbf{B}_{1}-\mathbf{B}_{2}\right)=\mathbf{0}
\end{gathered}
$$

After the vector calculation of (19) - (21), the following equations can be obtained as:

$$
\begin{aligned}
& H_{1 \theta}(r, \theta, \varepsilon) \\
& -\left.\frac{\varepsilon}{r} \sin (\theta-\varphi) H_{1 r}(r, \theta, \varepsilon)\right|_{r=R_{r}+\varepsilon \cos (\theta-\varphi)}=0 \\
& H_{1 \theta}(r, \theta, \varepsilon)-H_{2 \theta}(r, \theta, \varepsilon)-\frac{\varepsilon}{r} \sin (\theta-\varphi) \\
& \times\left.\left[H_{1 r}(r, \theta, \varepsilon)-H_{2 r}(r, \theta, \varepsilon)\right]\right|_{r=R_{m}+\varepsilon \cos (\theta-\varphi)}=0 \\
& B_{1 r}(r, \theta, \varepsilon)-B_{2 r}(r, \theta, \varepsilon)+\frac{\varepsilon}{r} \sin (\theta-\varphi) \\
& \times\left.\left[B_{1 \theta}(r, \theta, \varepsilon)-B_{2 \theta}(r, \theta, \varepsilon)\right]\right|_{r=R_{m}+\varepsilon \cos (\theta-\varphi)}=0
\end{aligned}
$$

By applying Eq. (3) into (22) and using Taylor series expansion at $r=R_{r}$ and $R_{m}$, the specific first-order boundary conditions can be obtained as:

$$
\begin{aligned}
& \left.\frac{\partial A_{z 1}^{(1)}}{\partial r}\right|_{r=R_{r}}=-\cos (\theta-\varphi) \frac{\partial^{2} A_{Z 1}^{(0)}}{\partial r^{2}} \\
& -\left.\sin (\theta-\varphi)\left(\frac{1}{r^{2}} \frac{\partial A_{z 1}^{(0)}}{\partial \theta}-\frac{1}{r} \mu_{0} M_{r}\right)\right|_{r=R_{r}} \\
& \frac{\partial A_{z 1}^{(1)}}{\partial r}-\left.\mu_{r} \frac{\partial A_{z 2}^{(1)}}{\partial r}\right|_{r=R_{m}}=-\cos (\theta-\phi)\left(\frac{\partial^{2} A_{z 1}^{(0)}}{\partial r^{2}}-\mu_{r} \frac{\partial^{2} A_{z 2}^{(0)}}{\partial r^{2}}\right) \\
& -\left.\sin (\theta-\phi)\left(\frac{1}{r^{2}} \frac{\partial A_{z 1}^{(0)}}{\partial \theta}-\frac{1}{r^{2}} \mu_{r} \frac{\partial A_{z 2}^{(0)}}{\partial \theta}-\frac{1}{r} \mu_{0} M_{r}\right)\right|_{r=R_{m}}
\end{aligned}
$$

$$
\begin{gathered}
\left.\frac{1}{r}\left(\frac{\partial A_{z 1}^{(1)}}{\partial \theta}-\frac{\partial A_{z 2}^{(1)}}{\partial \theta}\right)\right|_{r=R_{m}}= \\
\cos (\theta-\phi)\left(\frac{1}{r^{2}} \frac{\partial A_{z 1}^{(0)}}{\partial \theta}-\frac{1}{r^{2}} \frac{\partial A_{z 2}^{(0)}}{\partial \theta}-\frac{1}{r} \frac{\partial^{2} A_{z 1}^{(0)}}{\partial r \partial \theta}+\frac{1}{r} \frac{\partial^{2} A_{z 2}^{(0)}}{\partial r \partial \theta}\right) \\
+\left.\sin (\theta-\phi)\left(\frac{1}{r} \frac{\partial A_{z 1}^{(0)}}{\partial r}-\frac{1}{r} \frac{\partial A_{z 2}^{(0)}}{\partial r}\right)\right|_{r=R_{m}}
\end{gathered}
$$

where $A_{\mathrm{z} 1}{ }^{(0)}$ and $A_{\mathrm{z} 2}{ }^{(0)}$ are the zeroth-order magnetic vector potentials whose expressions have been fully discussed in [20].

Substituting the expressions of the zeroth- and first-order magnetic vector potentials to the first-order boundary condition (25), the following equations can be derived as:

$$
\begin{aligned}
& \sum_{k}\left(k R_{r} G_{1} A_{1}^{(1)}-k R_{r} B_{1}^{(1)}\right) \cos (k \theta) \\
+ & \sum_{k}\left(k R_{r} G_{1} C_{1}^{(1)}-k R_{r} D_{1}^{(1)}\right) \sin (k \theta) \\
= & \cos (\theta-\phi)\left[\sum_{k} P_{11}(k) \cos (k \theta)+\sum_{k} Q_{11}(k) \sin (k \theta)\right] \\
+ & \sin (\theta-\phi)\left[\sum_{k} P_{12}(k) \sin (k \theta)+\sum_{k} Q_{12}(k) \cos (k \theta)\right]
\end{aligned}
$$

where

$$
\begin{gathered}
P_{11}(k)=-2 k^{2} G_{1} A_{1}^{(0)}-\frac{\mu_{0} R_{r} k}{1-k} M_{r s k}+\frac{\mu_{0} R_{r} k^{2}}{1-k} M_{\theta c k} \\
P_{12}(k)=2 k G_{1} A_{1}^{(0)}+\frac{\mu_{0} R_{r} k}{1-k} M_{r s k}-\frac{\mu_{0} R_{r} k}{1-k} M_{\theta c k} \\
Q_{11}(k)=-2 k^{2} G_{1} C_{1}^{(0)}+\frac{\mu_{0} R_{r} k}{1-k} M_{r c k}+\frac{\mu_{0} R_{r} k^{2}}{1-k} M_{\theta s k} \\
Q_{12}(k)=-2 k G_{1} C_{1}^{(0)}+\frac{\mu_{0} R_{r} k}{1-k} M_{r c k}+\frac{\mu_{0} R_{r} k}{1-k} M_{\theta s k} \\
G_{1}=\left(R_{r} / R_{m}\right)^{k}
\end{gathered}
$$

where $A_{1}{ }^{(0)}$ and $C_{1}{ }^{(0)}$ are the integration constants of the zeroth magnetic vector potential in the PM region [20].

Similarly, according to the boundary condition (26), the following equation can be obtained as:

$$
\begin{aligned}
& \sum_{k} \cos (k \theta) \times {\left[-k R_{m} A_{1}^{(1)}+k R_{m} G_{1} B_{1}^{(1)}\right.} \\
&\left.+\mu_{r} k R_{m} G_{2} A_{2}^{(1)}-\mu_{r} k R_{m} B_{2}^{(1)}\right] \\
&+\sum_{k} \sin (k \theta) \times[-k R_{m} C_{1}^{(1)}+k R_{m} G_{1} D_{1}^{(1)} \\
&\left.+\mu_{r} k R_{m} G_{2} C_{2}^{(1)}-\mu_{r} k R_{m} D_{2}^{(1)}\right] \\
&=\sum_{k} \cos (\theta-\phi) \times\left[P_{21}(k) \cos (k \theta)+Q_{21}(k) \sin (k \theta)\right] \\
&+\sum_{k} \sin (\theta-\phi) \times\left[P_{22}(k) \sin (k \theta)+Q_{22}(k) \cos (k \theta)\right]
\end{aligned}
$$

where 


$$
\begin{aligned}
& P_{21}(k)=\left[k(k-1)+k(k+1) G_{1}^{2}\right] A_{1}^{(0)}-k(k-1) G_{2} A_{2}^{(0)} \\
&-k(k+1) B_{2}^{(0)} \\
&+\frac{k}{1-k} \mu_{0} R_{r} G_{1} M_{r s k}-\frac{k^{2}}{1-k} \mu_{0} R_{r} G_{1} M_{\theta c k} \\
& P_{22}(k)=-k\left(1+G_{1}^{2}\right) A_{1}^{(0)}+k G_{2} A_{2}^{(0)}+k B_{2}^{(0)} \\
&-\frac{k}{1-k^{2}} \mu_{0}\left(R_{r} G_{1}+k R_{m}\right) M_{r s k} \\
&+\frac{k}{1-k^{2}} \mu_{0}\left(k R_{r} G_{1}+R_{m}\right) M_{\theta c k} \\
& Q_{21}(k)=\left[k(k-1)+k(k+1) G_{1}^{2}\right] C_{1}^{(0)}-k(k-1) G_{2} C_{2}^{(0)} \\
&-k(k+1) D_{2}^{(0)} \\
&-\frac{\mu_{0} R_{r} k}{1-k} G_{1} M_{r c k}-\frac{\mu_{0} R_{r} k^{2}}{1-k} G_{1} M_{\theta s k} \\
& Q_{22}(k)=k\left(1+G_{1}^{2}\right) C_{1}^{(0)}-k G_{2} C_{2}^{(0)}-k D_{2}^{(0)} \\
&-\frac{\mu_{0} k}{1-k^{2}}\left(R_{r} G_{1}+k R_{m}\right) M_{r c k} \\
&- \frac{\mu_{0} k}{1-k^{2}}\left(k R_{r} G_{1}+R_{m}\right) M_{\theta s k} \\
& G_{2}=\left(R_{m} / R_{s}\right)^{k}
\end{aligned}
$$

where $A_{2}{ }^{(0)}, B_{2}{ }^{(0)}, C_{2}{ }^{(0)}$ and $D_{2}{ }^{(0)}$ are the integration constants of zeroth magnetic vector potential in the air gap region [20].

Finally, considering boundary condition (27), the following equation can be derived as:

$$
\begin{aligned}
& \sum_{k} \cos (k \theta) \times {\left[k R_{m} C_{1}^{(1)}+k R_{m} G_{1} D_{1}^{(1)}\right.} \\
&\left.-k R_{m} G_{2} C_{2}^{(1)}-k R_{m} D_{2}^{(1)}\right] \\
&+\sum_{k} \sin (k \theta) \times {\left[-k R_{m} A_{1}^{(1)}-k R_{m} G_{1} B_{1}^{(1)}\right.} \\
&+\left.k R_{m} G_{2} A_{2}^{(1)}+k R_{m} B_{2}^{(1)}\right] \\
&=\sum_{k} \cos (\theta-\phi) \times\left[P_{31}(k) \cos (k \theta)+Q_{31}(k) \sin (k \theta)\right] \\
&+\sum_{k} \sin (\theta-\phi) \times\left[P_{32}(k) \sin (k \theta)+Q_{32}(k) \cos (k \theta)\right]
\end{aligned}
$$

where

$$
\begin{aligned}
P_{31}(k) & =\left[-k(k-1)+k(k+1) G_{1}^{2}\right] C_{1}^{(0)}+k(k-1) G_{2} C_{2}^{(0)} \\
& -k(k+1) D_{2}^{(0)} \\
& -\frac{k}{1-k} \mu_{0} R_{r} G_{1} M_{r c k}-\frac{k^{2}}{1-k} \mu_{0} R_{r} G_{1} M_{\theta s k} \\
P_{32}(k)= & k\left(1-G_{1}^{2}\right) C_{1}^{(0)}-k G_{2} C_{2}^{(0)}+k D_{2}^{(0)} \\
+ & \frac{k}{1-k^{2}} \mu_{0}\left(R_{r} G_{1}-R_{m}\right) M_{r c k} \\
& -\frac{1}{1-k^{2}} \mu_{0}\left(-k^{2} R_{r} G_{1}+R_{m}\right) M_{\theta s k}
\end{aligned}
$$

$$
\begin{aligned}
Q_{31}(k) & =\left[k(k-1)-k(k+1) G_{1}^{2}\right] A_{1}^{(0)} \\
& -k(k-1) G_{2} A_{2}^{(0)}+k(k+1) B_{2}^{(0)} \\
& -\frac{k}{1-k} \mu_{0} R_{r} G_{1} M_{r s k}-\frac{k^{2}}{1-k} \mu_{0} R_{r} G_{1} M_{\theta c k} \\
Q_{32}(k) & =k\left(1-G_{1}^{2}\right) A_{1}^{(0)}-k G_{2} A_{2}^{(0)}+k B_{2}^{(0)} \\
& -\frac{k}{1-k^{2}} \mu_{0}\left(R_{r} G_{1}-R_{m}\right) M_{r s k} \\
& -\frac{1}{1-k^{2}} \mu_{0}\left(-k^{2} R_{r} G_{1}+R_{m}\right) M_{\theta c k}
\end{aligned}
$$

Besides (28), (34) and (40), other boundary condition equations can be obtained by considering the interface between the air gap and stator slot openings and the interface sandwiched by the stator slot openings and slots. All of these equations can be solved as a whole by transforming them in the matrix form [19-21]. Accordingly, all the integration coefficients, viz. $A_{1}^{(1)}-D_{4 i}{ }^{(1)}$ in expression (8)-(11), can be determined and hence the complete expressions of the magnetic vector potentials in all subdomains are obtained, which lays a basis for the prediction of flux densities, flux linkages and torque in the machines.

\section{FE Validation and Investigation}

The FEM models of the $8 \mathrm{p}-12 \mathrm{~s}$ and $8 \mathrm{p}-9 \mathrm{~s}$ SPM machines with rotor eccentricity are adopted to provide verification of the analytical results, and the main model parameters are shown in Table 1. In this paper, the static eccentricity is set as that the rotor is shifted from the stator centre to the right by $0.5 \mathrm{~mm}$ along the $r$-axis, viz. the static eccentricity distance and angle are $0.5 \mathrm{~mm}$ and 0 degrees, respectively. It is also the initial position for the dynamic eccentricity. Considering the no-load operation as one particular case of the load conditions, this paper is mainly focused on the rotor eccentricity analysis of the SPM machines with armature reaction, and corresponding results about no-load conditions can be obtained by simply

Table 1. The main parameters of the machine models

\begin{tabular}{ccc}
\hline Parameters & $\begin{array}{c}\text { Model I } \\
(8 \mathrm{p}-12 \mathrm{~s})\end{array}$ & $\begin{array}{c}\text { Model II } \\
(8 \mathrm{p}-9 \mathrm{~s})\end{array}$ \\
\hline Pole number & 8 & 8 \\
Slot number & 12 & 9 \\
Inner radius of rotor core $(\mathrm{mm})$ & 15 & 15 \\
Outer radius of rotor core $(\mathrm{mm})$ & 23.85 & 23.85 \\
PM length (mm) & 3 & 3 \\
Inner radius of air gap (mm) & 26.85 & 26.85 \\
Outer radius of air gap (mm) & 27.85 & 27.85 \\
Slot opening width angle & $6.3^{\circ}$ & $7.8^{\circ}$ \\
Slot width angle & $13.82^{\circ}$ & $18.24^{\circ}$ \\
Rated current (A) & 10 & 10 \\
Winding turns per phase & 34 & 34 \\
Static eccentricity distance $(\mathrm{mm})$ & 0.5 & 0.5 \\
Static eccentricity angle & $0^{\circ}$ & $0^{\circ}$ \\
\hline
\end{tabular}


lowering the winding currents to zero. The equal potential distributions of the two adopted models with static eccentricity and armature reaction are shown in Fig. 2.

\subsection{Air gap flux density}

As shown in Fig. $3 a$ and $b$, for the 8p-12s machine with static eccentricity and symmetric load, both of the radial and circumferential components of flux density predicted by the analytical model agree very well with the FEM results. For better clarification about the effect of rotor eccentricity, the flux densities corresponding to the normal rotor have also been presented in the figures.

Obviously, the rotor eccentricity has significant effect on the radial component but little effect on the tangential component of the air gap flux density. This can be explained by the nonuniformity of the air gap caused by the rotor eccentricity. In the eccentric model, the maximum air gap length is $1.5 \mathrm{~mm}$, which is three times of the minimum air gap length. As shown by Fig. 3(a), the amplitude of the radial flux density is $1.35 \mathrm{~T}$ at the point of the minimum air gap length, and reduces to $1.0 \mathrm{~T}$ at the point corresponding to the maximum air gap length.

Fig. $3 c$ displays the harmonics of the radial component

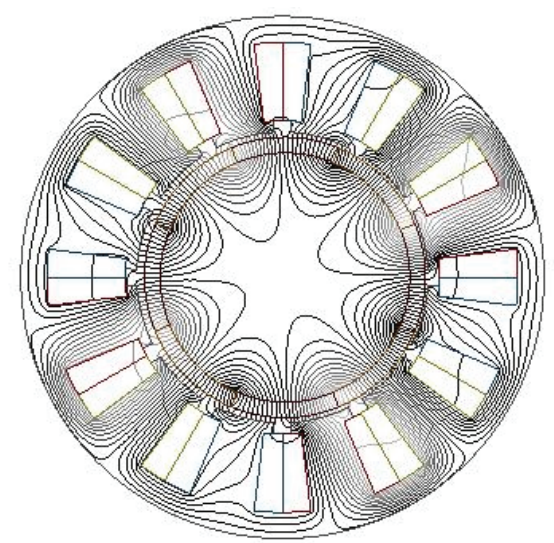

(a) The $8 \mathrm{p}-12 \mathrm{~s}$ model with $\mathrm{i}_{\mathrm{A}}=-\mathrm{i}_{\mathrm{B}}=-8.66 \mathrm{~A}, \mathrm{i}_{\mathrm{C}}=0 \mathrm{~A}$

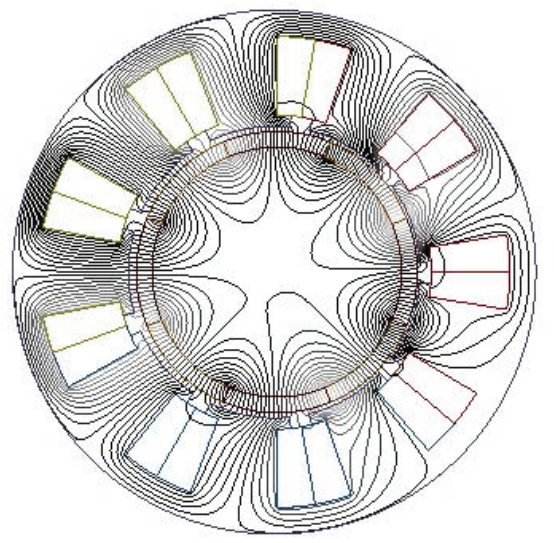

(b) The $8 \mathrm{p}-9 \mathrm{~s}$ model with $\mathrm{i}_{\mathrm{B}}=\mathrm{i}_{\mathrm{C}}=5 \mathrm{~A}, \mathrm{i}_{\mathrm{A}}=-10 \mathrm{~A}$

Fig. 2. The equal potential distributions of air gap flux density. For the normal rotor, the orders of the harmonics are odd times of the pole-pairs number, while for the eccentric one, the radial component has more specific harmonics caused by the effect of rotor eccentricity. The orders of these harmonics are bigger or smaller than the normal orders by 1 .

For the $8 \mathrm{p}-9 \mathrm{~s}$ SPM machine, similar conclusions can be obtained compared to the 8p-12s SPM machine.

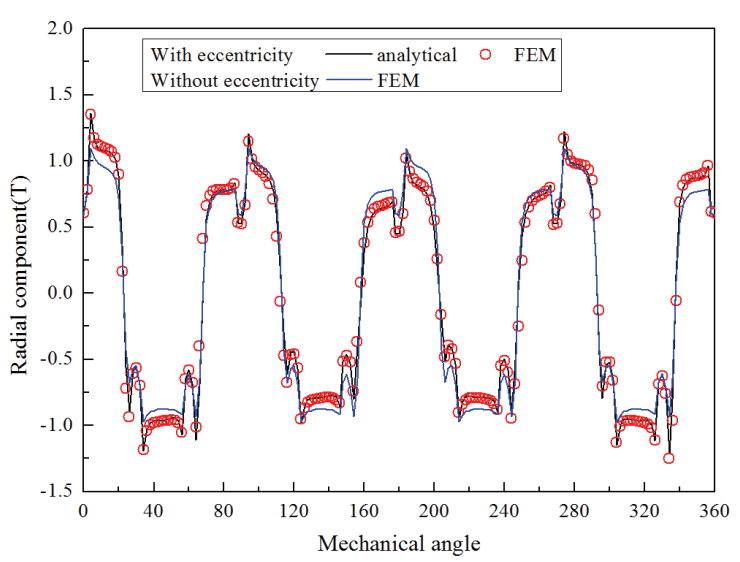

(a) The radial component

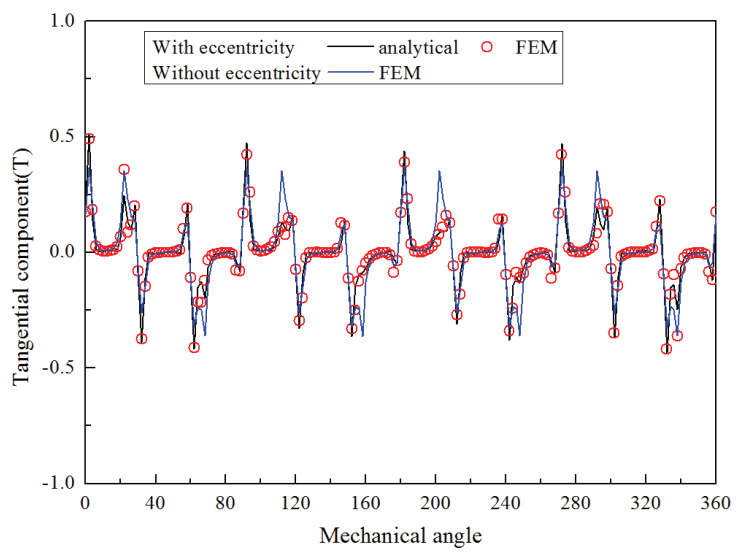

(b) The tangential component

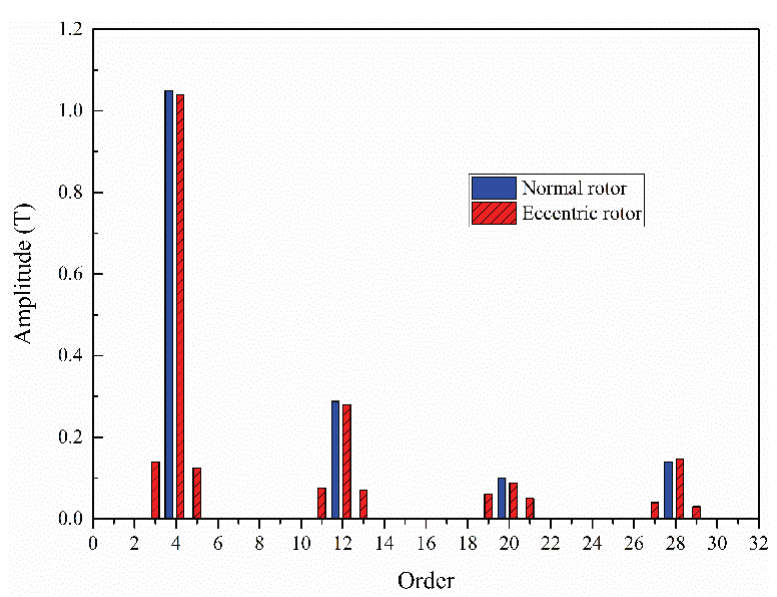

(c) The harmonics

Fig. 3. The air gap flux density (8p-12s) 


\subsection{Flux linkage and torque with static eccentricity}

Fig. 4 shows the results comparisons between the FEM and analytical method for the flux linkages of the $8 p-12 s$ and $8 p-9$ s SPM machine with static eccentricity and symmetric load. As shown by Fig. 4(a), for the 8p$12 \mathrm{~s}$ machine, the flux linkage amplitudes of the four coils constituting each phase are asymmetric and vary widely. This can be explained by the difference in lengths of the air gap adjacent to the coils. Taking phase A as an example, in the decreasing sequence, the amplitudes of coil A1, A4, A2 and A3 are 0.026, 0.023, 0.021 and $0.019 \mathrm{~Wb}$, respectively. The successive decrease is caused by the incremental air gap lengths corresponding to the four coils. However, as the length of the air gap adjacent to each coil remains constant during the rotation, the amplitude of the flux linkage also remains unchanged.

As shown by Fig. 4(b), compared to the coils whose amplitudes are different with each other, the three-phase flux linkages of the $8 \mathrm{p}-12 \mathrm{~s}$ machine with static eccentricity are symmetrical. The main reason is that the coils of each phase are uniformly distributed along the circumference, and the effects of rotor eccentricity are counteracted by the balanced distribution.

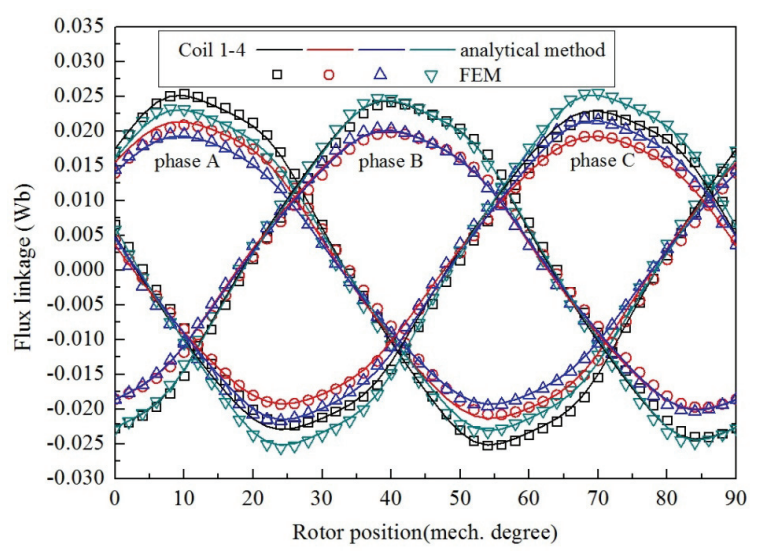

(a) The coil flux linkage (8p-12s)

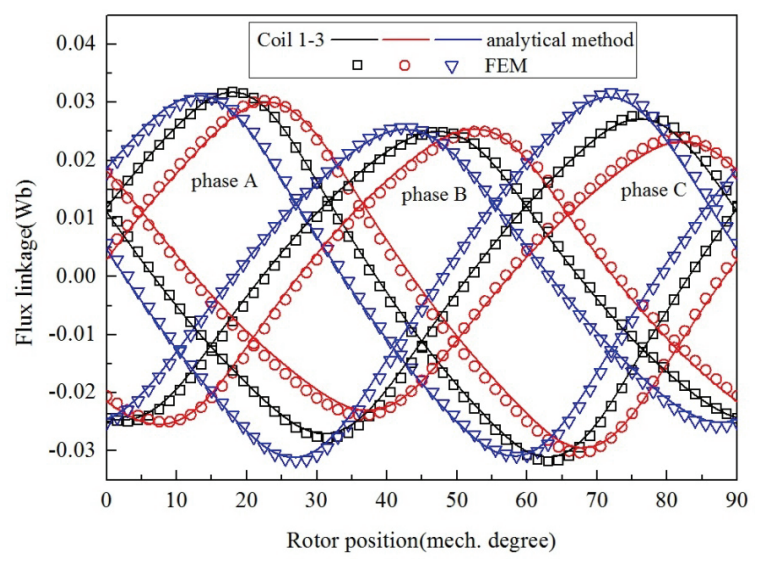

(c) The coil flux linkage (8p-9s)
Results comparison in Fig. 4(c) also displays the amplitude difference of the coils. However, as shown by Fig. 4(d), the three-phase flux linkages are not symmetrical in the $8 \mathrm{p}-9 \mathrm{~s}$ model. The reason is that the coils belonging to each phase are cluster together. For instance, all three coils of phase A are nearest to the minimum air gap in this model, so the flux linkage of phase A has the maximum amplitude compared with the other two phases. But for each single phase, the amplitude remains constant with the rotation.

Fig. 5 shows the effect of static eccentricity on the total and cogging torque. Results comparisons between the normal and static eccentricity torque illustrate that the static eccentricity has little effect on the cogging torque [12]. The total torque is the sum of the cogging and electromagnetic torque, and the electromagnetic torque can be expressed as:

$$
T_{e}=\frac{e_{a} i_{a}+e_{b} i_{b}+e_{c} i_{c}}{\omega_{r}}
$$

where $e_{a}, e_{b}, e_{c}, i_{a}, i_{b}$, and $i_{c}$ are the three-phase backEMFs and currents. As the static eccentricity has little effect on the back-EMF and cogging torque, so does the

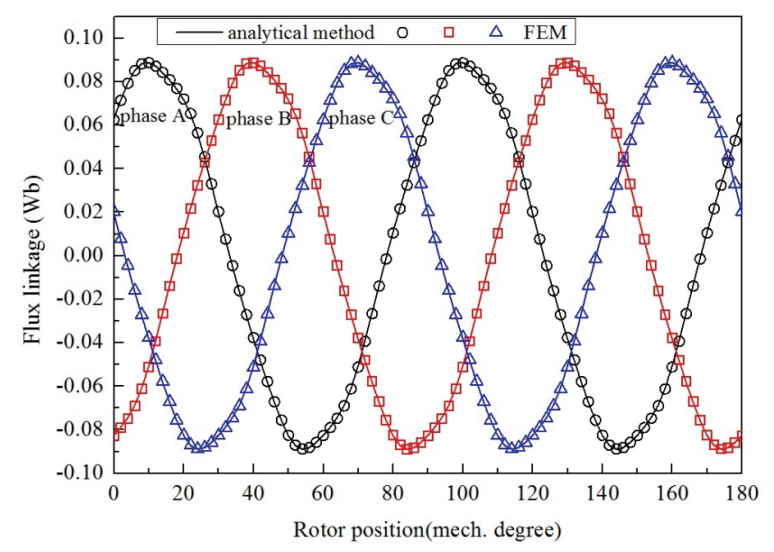

(b) The phase flux linkage (8p-12s)

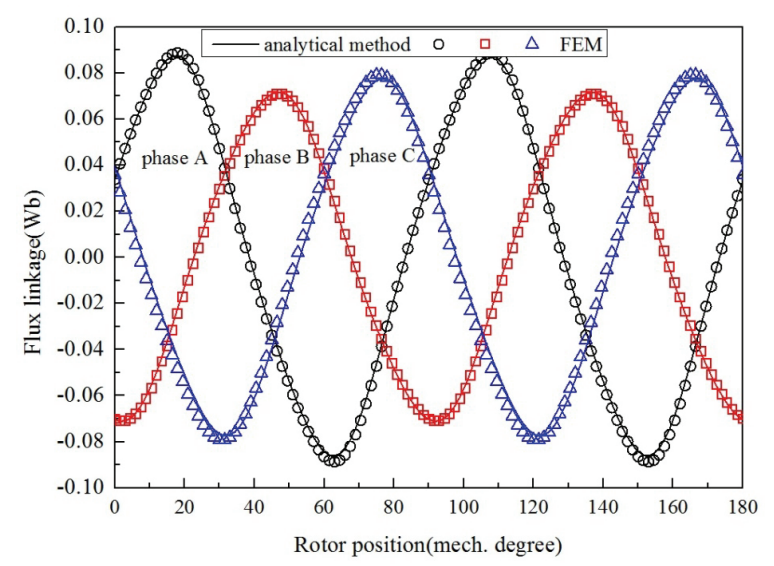

(d) The phase flux linkage $(8 p-9 s)$

Fig. 4. The flux linkage comparison for static eccentricity 
total torque.

For the $8 \mathrm{p}-9 \mathrm{~s}$ SPM machine, the static eccentricity has great effect on the cogging torque, and both the amplitude and period are changed [12]. As the static eccentricity has great effect on the back-EMFs, the electromagnetic torque and the total torque also change much compared with the machine with normal rotor.

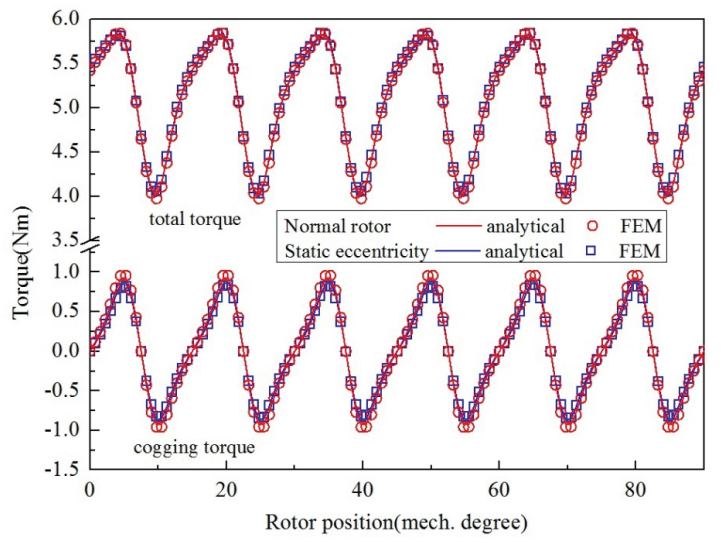

(a) The total and cogging torque (8p-12s)

\subsection{Flux linkage and torque with dynamic eccentricity}

Fig. 6(a) and (b) show the results comparisons of flux linkages for $8 \mathrm{p}-12 \mathrm{~s}$ SPM machine with the dynamic eccentricity and symmetric load. When the position of minimum air gap changes with the rotation, the minimum air gap interacts with all the coils during the rotation.

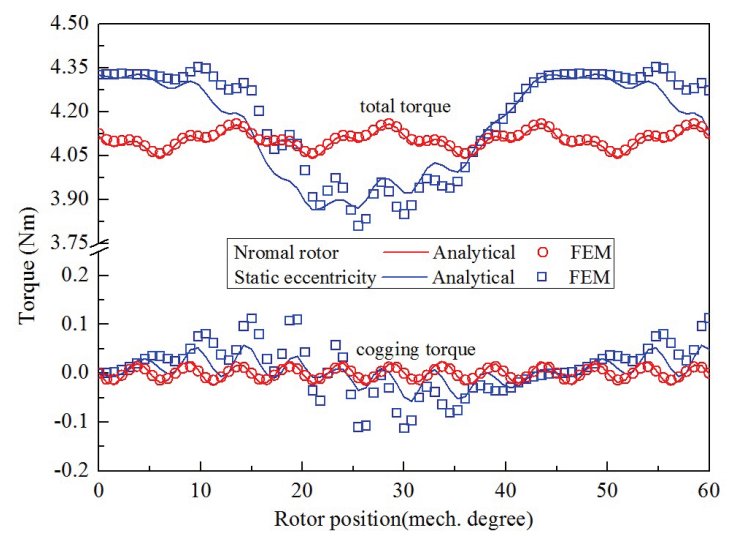

(b) The total and cogging torque $(8 \mathrm{p}-9 \mathrm{~s})$

Fig. 5. The torque comparison for static eccentricity

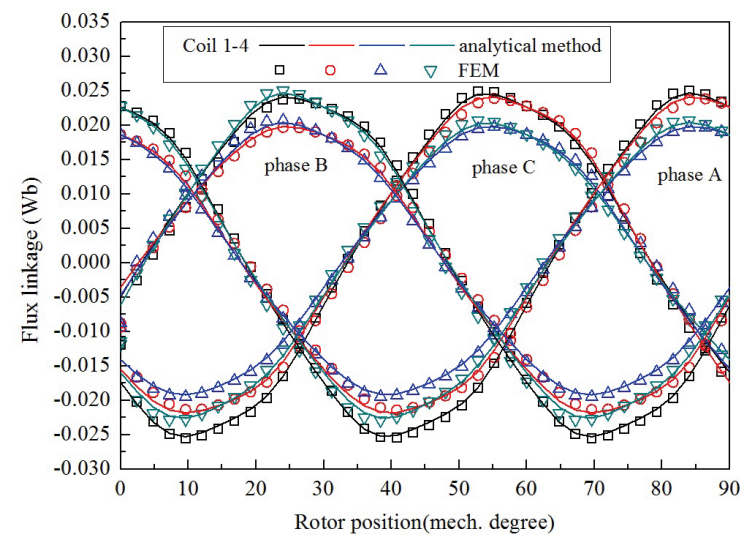

(a) The coil flux linkage (8p-12s)

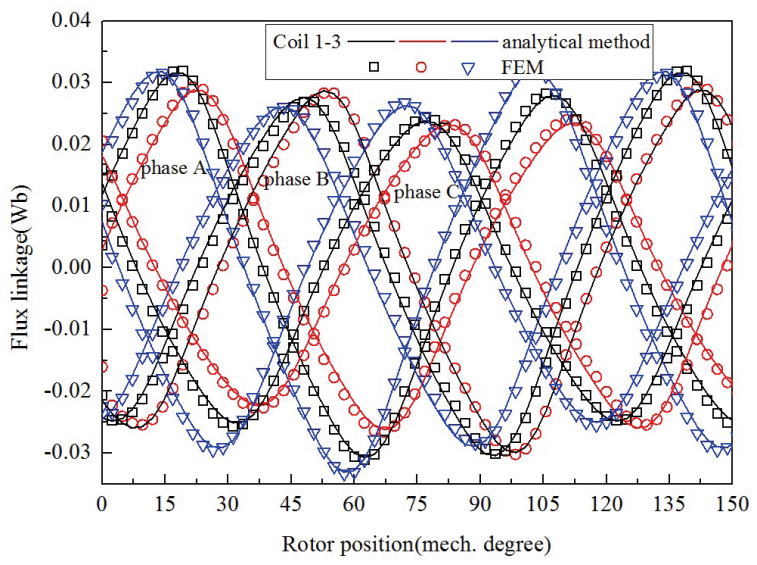

(c) The coil flux linkage (8p-9s)

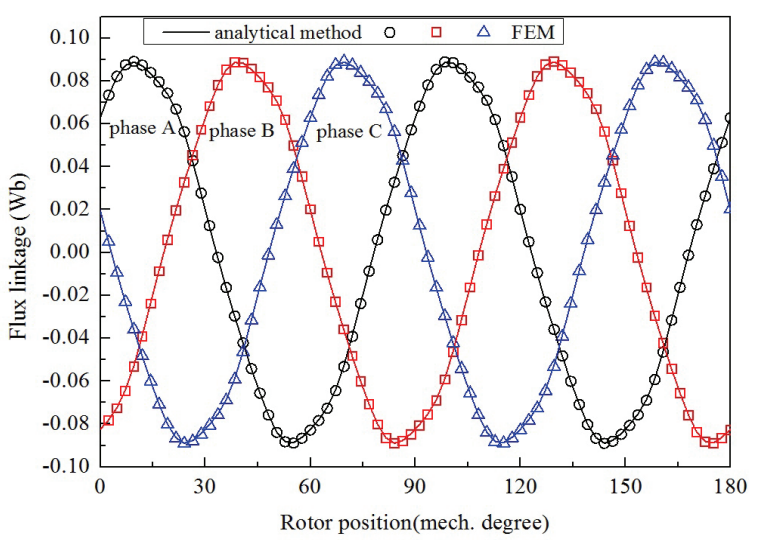

(b) The phase flux linkage (8p-12s)

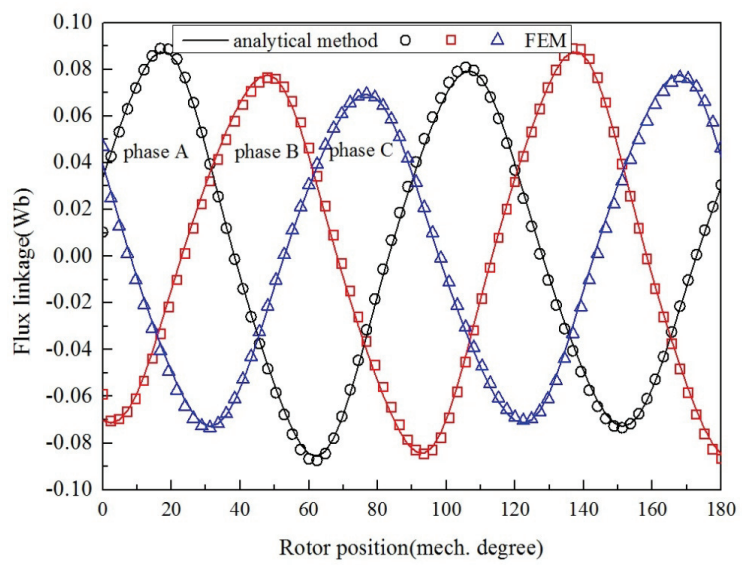

(d) The phase flux linkage (8p-9s)

Fig. 6. The flux linkage comparison for dynamic eccentricity 
The flux linkages of all the coils have the same waveform except for the phase angle. Taking phase A as an example, the flux linkage of coil A1 and A2 have the same waveforms, but the flux linkage of A2 lags that of A1 about 90 degrees. For the flux linkage of the single coil, the amplitude changes with the rotation. The phase flux linkage is the sum of those of the coils, so the flux linkages

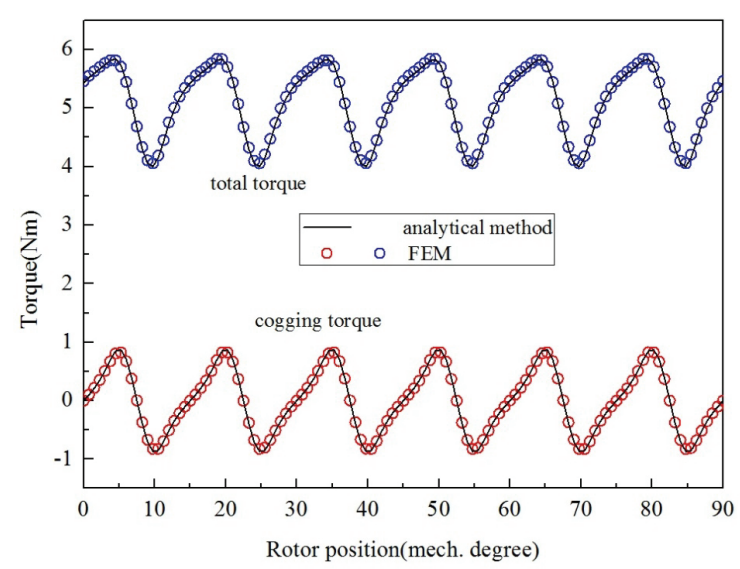

(a) The total and cogging torque (8p-12s) of the three-phase are symmetrical.

Fig. 6(c) and d show the effect of dynamic eccentricity on the flux linkages of the 8p-9s SPM machine. For the flux linkage of every single coil, the amplitude changes with the rotation, but the flux linkages of all the coils have the same waveform except for the phase angle. The phase flux linkages have the same waveforms, and the amplitudes

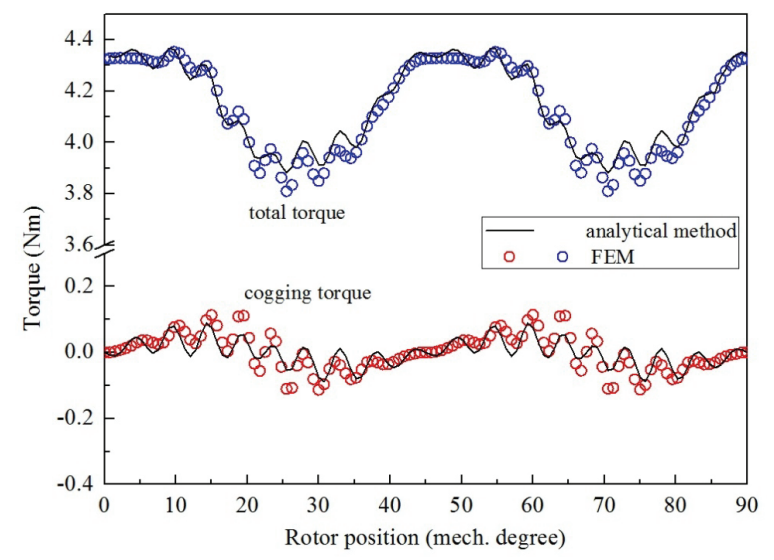

(b) The total and cogging torque $(8 \mathrm{p}-9 \mathrm{~s})$

Fig. 7. The torque comparison for dynamic eccentricity

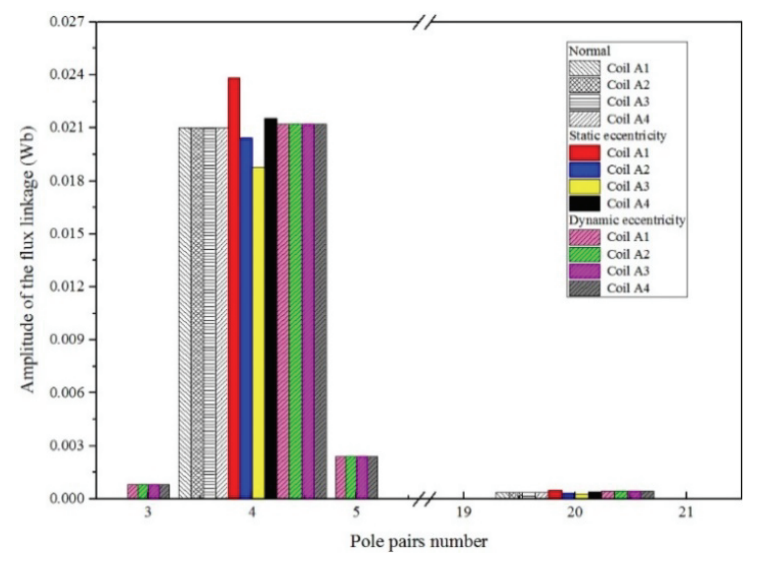

(a) The harmonics of coil flux linkages of phase A (8p-12s)

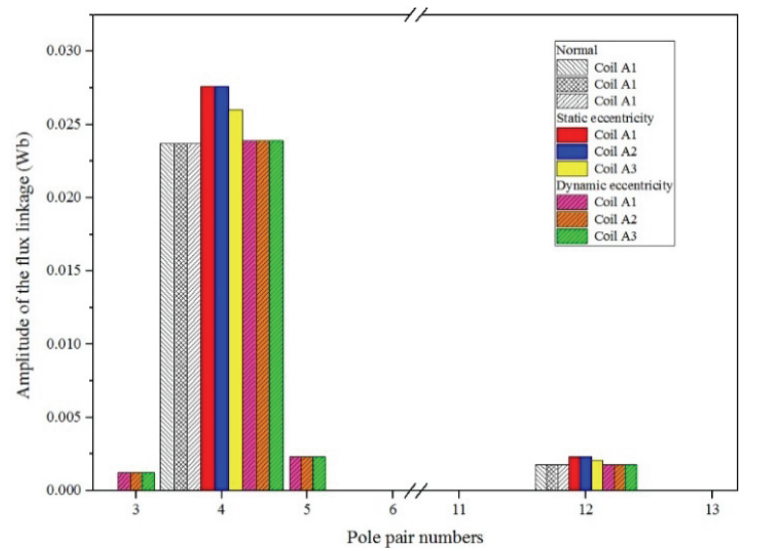

(c) The harmonics of coil flux linkages of phase A (8p-9s)

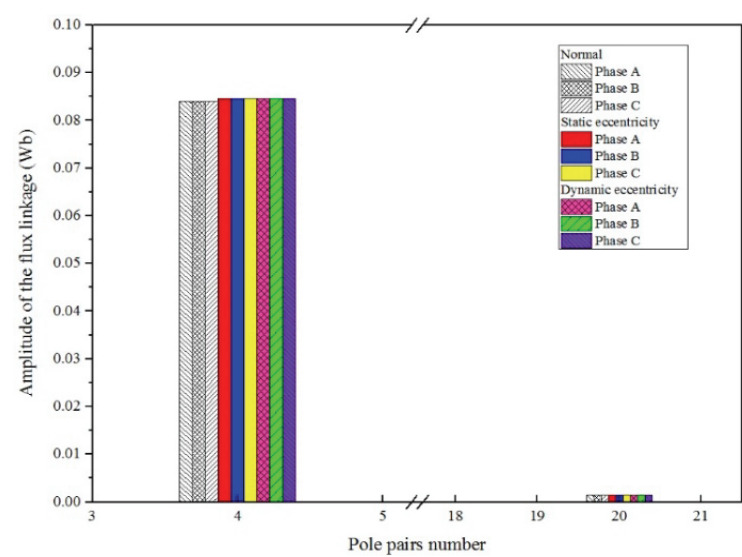

(b) The harmonics of phase flux linkages (8p-12s)

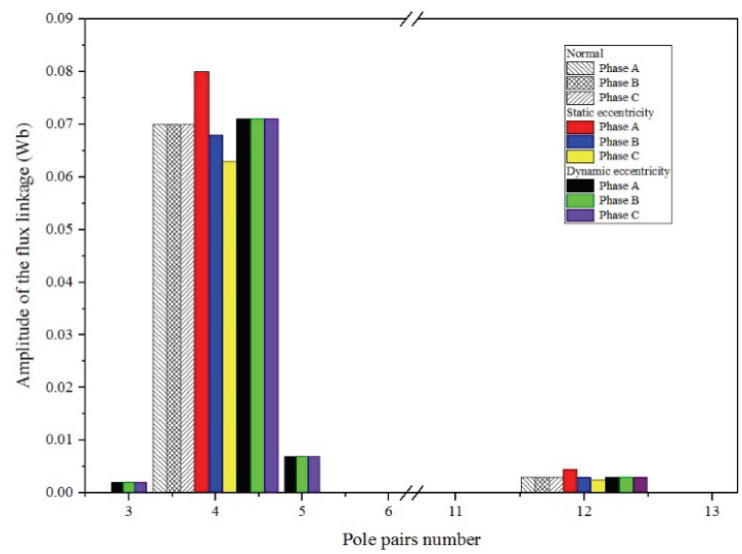

(d) The harmonics of phase flux linkages (8p-9s)

Fig. 8. The harmonics of flux linkages 
change with the rotation. The three-phase flux linkages are not symmetrical for the 8p-9s SPM machine.

Fig. 7 shows the effect of dynamic eccentricity on the total and cogging torque. Just as the static eccentricity, the dynamic eccentricity has little effect on the cogging torque and electromagnetic torque for the 8p-12s SPM machine. But it has great effect on the cogging torque and electromagnetic torque for the $8 \mathrm{p}-9 \mathrm{~s}$ SPM machine.

The accuracy of the analytical method has been well validated by the results comparisons shown by Fig. 3 to 7 , followed by the corresponding discussions. For a further analysis about the eccentricity effect, Fig. 8 compares the harmonic amplitudes of the coil and phase flux linkages under load condition in the $8 \mathrm{p}-12 \mathrm{~s}$ and $8 \mathrm{p}-9 \mathrm{~s}$ SPM machines with normal or eccentric rotors.

For the coil flux linkages of the 8p-12s SPM machine with static eccentricity, although no additional harmonics are introduced, the fundamental components have different amplitudes because the lengths of air gap adjacent to the coils are different. As the fundamental components of coil flux linkages have the same phase angles, the amplitude of the phase flux linkage is the summation of the coil flux linkages. Because the four coils are uniformly distributed along the circumference, the effects of the eccentricity on the flux linkages of coils A1 and A3 (A2 and A4) are in reverse. For the phase flux linkage, the static eccentricity has little effect.

The dynamic eccentricity has little effect on the fundamental components of coil flux linkage, but additional harmonics with 3 and 5-pole pairs are introduced. For the harmonics with 3 or 5-pole pair for each coil, the amplitudes are the same and the phase angles difference are 90 degrees, so both harmonics are offset in the phase flux linkage. Then the dynamic eccentricity also has little effect on the phase flux linkage.

For the static eccentricity, the fundamental components of coil flux linkages (coils A1-A3) have different amplitudes and no additional harmonics are introduced for the $8 \mathrm{p}-9 \mathrm{~s}$ SPM machine. As the three coils of phase A are adjacent to the minimum air gap, the flux linkage of phase $\mathrm{A}$ has the maximum value among the three phases and the threephase flux linkages are not symmetrical.

For the dynamic eccentricity, the fundamental components of coil flux linkages change little compared with the SPM machine with normal rotor, but additional harmonics with 3 and 5-pole pair are introduced. For the harmonics with 3 or 5-pole pair, the amplitudes are the same with each other, and the phase angles difference are 60 and 20 degrees respectively, so both the harmonics can be found in the phase flux linkage.

\section{Results Error Analysis}

In this paper, all the physical quantities validated by the FEM results are calculated based on the magnetic vector

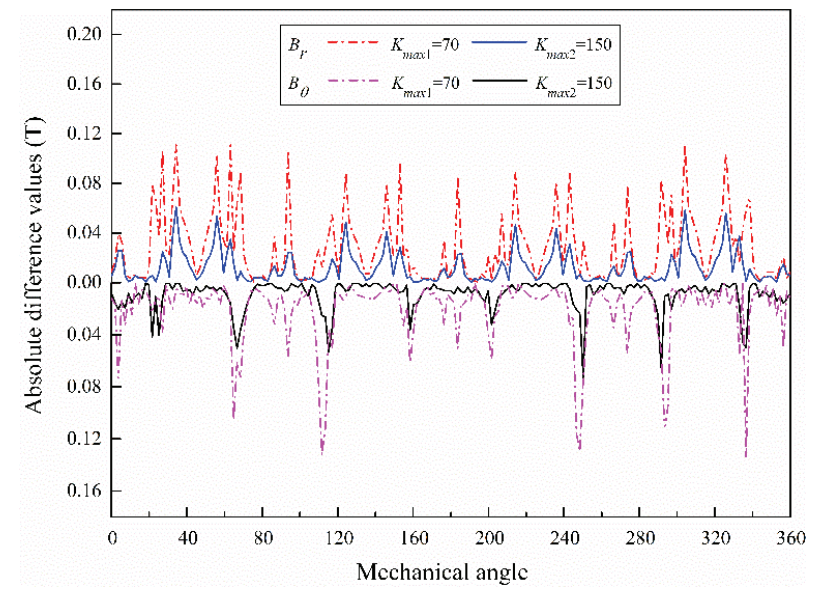

Fig. 9. The absolute difference values between the analytical and the FEM results

potential, viz. $A_{z}$, which can be expressed in the Fourier series expansion. In the practical situation, the harmonic order of the expansion should range from one to positive infinity, whereas in the actual calculation process, the highest order can not reach positive infinity. The components corresponding to the omitted orders are the main reason for the calculation error, which indicates that the results precision can be improved with the enlargement of the maximum harmonic order. However, the maximum order can not be set as an excessively large value which can greatly exaggerate the dimension of solution matrix followed by the reduced calculation speed.

The radial and the tangential component of flux density, viz. $\mathrm{B}_{\mathrm{r}}$ and $\mathrm{B}_{\theta}$, can be obtained by the partial derivations of $\mathrm{A}_{\mathrm{z}}$. Consequently, the absolute values of differences between the analytical and the FEM results of $B_{r}$ and $B_{\theta}$ can be reasonably treated as the quantification of calculation accuracy. In Fig. 9, the absolute difference values of $\mathrm{B}_{\mathrm{r}}$ and $\mathrm{B}_{\theta}$ in the $8 \mathrm{p}-12 \mathrm{~s}$ machine with static eccentricity and armature reaction are displayed and two selections of the highest harmonic order, including $\mathrm{K}_{\max 1}=70$ and $\mathrm{K}_{\max 2}=150$, are respectively applied in the analytical method. Obviously, the calculation error is distinctly reduced with the increment of the highest harmonic order. For $\mathrm{K}_{\max 1}=70$, the maximum error values of $B_{r}$ and $B_{\theta}$ are respectively 0.11 and $0.13 \mathrm{~T}$, while for $\mathrm{K}_{\max 2}=150$, the counterpart values reduce to 0.06 and 0.07 $\mathrm{T}$.

\section{Conclusions}

The subdomain modelling with magnetic vector potential for the SPM machine is advised in this paper. The analytical results of the two machine models adopted for validation are in well agreement with the FEM predictions and the quantification of accuracy is also discussed by the results error analysis. Besides the well calculation 
precision, another prominent advantage of the analytical model is much less time-consuming compared to the FEM. To give an example for illustration, for the no-load flux linkage of 8p-12s SPM machine with static eccentricity, the computation time is about 35 seconds with the analytical model whereas the FEM takes about three minutes with a mesh of 19356 nodes and 35324 elements.

For the SPM machines with uniformly distributed phase windings (for example, $4 \mathrm{p}-12 \mathrm{~s}$ and $8 \mathrm{p}-12 \mathrm{~s}$ ), both the static and dynamic eccentricity have little effects on the phase flux linkage and torque. The main reason is that the different rotor eccentricity effects on the coil flux linkages are offset for the phase values. For the other specific pole and slot number combinations, in which the phase windings are not uniformly distributed (for example, $8 \mathrm{p}-9 \mathrm{~s}$ and $10 \mathrm{p}-9 \mathrm{~s}$ ), the effects of rotor eccentricity on phase flux linkage and torque are amplified.

Considering the utilization commonality possessed by the analytical method, the subdomain model proposed in this paper can be generally applied in the rotor eccentricity analysis about PM machines with different configurations and operating principles. In further investigations, the analytical results will be obtained through the subdomain method and provided as the quantitative analysis of field distributions and electromagnetic performance for variant PM machines.

\section{Acknowledgements}

This work was financially supported by the National Natural Science Foundation of China under grant 51107075 .

\section{References}

[1] Kyung-Tae Kim, Kwang-Suk Kim, Sang-Moon Hwang, Tae-Jong Kim, and Yoong-Ho Jung, "Comparison of magnetic forces for IPM and SPM motor with rotor eccentricity," IEEE Trans. Magn., vol. 37, no. 5, pp. 3448-3451, Sept. 2001.

[2] David G. Dorrell, Min-Fu Hsieh, YouGuang Guo, "Unbalanced magnet pull in large brushless rare-earth permanent magnet motors with rotor eccentricity," IEEE Trans. Magn., vol. 45, no. 10, pp. 4586-4589, Oct. 2009.

[3] Shintarou Araki, Toshinari Kondou, Akio Yamagiwa, "Analyses of electromagnetic forces of concentrated winding permanent magnet brushless motors with rotor eccentricity," International Conference on Electrical Machines and Systems, Tokyo, Japan, pp. 1-4, Nov. 2009.

[4] Min-Fu Hsieh, Yu-Han Yeh, "Rotor eccentricity effect on cogging torque of PM generators for small wind turbines," IEEE Trans. Magn., vol. 49, no. 5, pp.
1897-1900, May. 2013.

[5] Ji. L, R. Qu, Y. Cho, "Dynamic eccentricity in singlerotor single-stator axial flux permanent magnet synchronous machine with parallel path windings," IEEE Magnetics Conference, Beijing, China, pp. 1-1, May. 2015.

[6] Richard Perers, Urban Lundin, Mats Leijon, "Saturation effects on unbalanced magnetic pull in a hydroelectric generator with an eccentric rotor," IEEE Trans. Magn., vol. 43, no. 10, pp. 3884-3890, Oct. 2007.

[7] Hanafy Mahmoud, Nicola Bianchi, "Eccentricity in synchronous reluctance motors-Part I: analytical and finite-element models," IEEE Trans. Energy Conversion, vol.30, no. 2, pp. 745-753, June. 2015.

[8] Z. J. Liu, J. T. Li, M. A. Jabbar, "Prediction and analysis of magnetic forces in permanent magnet brushless dc motor with rotor eccentricity," Journal of Applied Physics, vol. 99, no. 8, pp. 1-3, Apr. 2006.

[9] J. T. Li, Z. J. Liu, L. H. A. Nay, "Effect of radial magnetic forces in permanent magnet motors with rotor eccentricity," IEEE Trans. Magn., vol. 43, no. 6, pp. 2525-2527, June. 2007.

[10] Jin-Po Wang, D. K. Lieu, "Magnetic lumped parameter modelling of rotor eccentricity in brushless permanent-magnet motors," IEEE Trans. Magn., vol. 35, no. 5, pp. 4226-4231, Sept. 1999.

[11] L. S. Stephens, M. A. Casemore, "Influence of stator slot geometry and rotor eccentricity on field distribution in cylindrical magnetic actuators," IEEE Trans. Magn., vol. 38, no. 2, pp. 1348-1356, Mar. 2002.

[12] Z. Q. Zhu, L. J. Wu, M. L. Mohd Jamil, "Influence of pole and slot number combinations on cogging torque in permanent-magnet machines with static and rotating eccentricities," IEEE Trans. Ind. Appl., vol. 50, no. 5, pp. 3265-3277, Sept/Oct. 2014.

[13] Daeyeon Kim, Myounggyu D. Noh, and Young-woo Park, "Unbalanced Magnetic Forces Due to Rotor Eccentricity in a Toroidally Wound BLDC Motor," IEEE Trans. Magn., vol. 52, no. 7, pp. 8203204, July. 2016.

[14] U. Kim, D. K. Lieu, "Magnetic field calculation in permanent magnet motors with rotor eccentricity: Without slotting effect," IEEE Trans. Magn., vol. 34, no. 4, pp. 2243-2252, July. 1998.

[15] U. Kim, D. K. Lieu, "Magnetic field calculation in permanent magnet motors with rotor eccentricity: With slotting effect considered," IEEE Trans. Magn., vol. 34, no. 4, pp. 2253-2266, July. 1998.

[16] Hao Qian, Hong Guo, Zhiyong Wu, Xiaofeng Ding, "Analytical solution for cogging torque in surfacemounted permanent-magnet motors with magnet imperfections and rotor eccentricity," IEEE Trans. Magn., vol. 50, no. 8, pp. 8201615, Aug. 2014.

[17] Jiajing Fu, Changsheng Zhu, "Subdomain model for 
predicting magnetic field in slotted surface mounted permanent-magnet machines with rotor eccentricity," IEEE Trans. Magn., vol. 48, no. 5, pp. 1906-1917, May. 2012.

[18] A. Rahideh, T. Korakianitis, "Analytical open-circuit magnetic field distribution of slotless brushless permanent-magnet machines with rotor eccentricity," IEEE Trans. Magn., vol. 47, no. 12, pp. 4791-4808, Dec. 2011.

[19] L. J. Wu, Z. Q. Zhu, D. Staton, M. Popescu, D. Hawkins, "Subdomain model for predicting armature reaction field of surface-mounted permanent-magnet machines accounting for tooth-tips," IEEE Trans. Magn., vol. 47, no. 4, pp. 812-822, Apr. 2011.

[20] L. J. Wu, Z. Q. Zhu, D. Staton, M. Popescu and D. Hawkins, "An improved subdomain model for predicting magnetic field of surface-mounted permanent magnet machines accounting for toothtips," IEEE Trans. Magn., vol. 47, no. 6, pp. 16931704, June. 2011.

[21] L. J. Wu, Z. Q. Zhu, D. Staton, M. Popescu and D. Hawkins, "Analytical prediction of electromagnetic performance of surface-mounted PM machines based on subdomain model accounting for tooth-tips," IET Electr. Power Appl., vol. 5, no. 7, pp. 597-609, Aug. 2011.

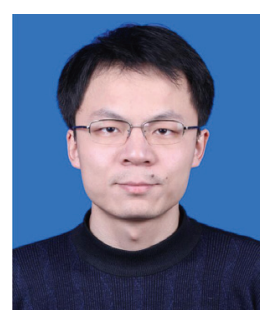

Bo Yan He received B.E. degree in electrical engineering from Shandong University, Jinan, China, in 2014. He is currently working toward the Ph.D. degree in Shandong University on electrical machinery and apparatus. His research interest is field calculation of the permanent magnet motors.

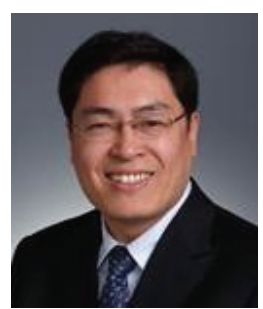

Xiuhe Wang He received the B.E. and M.E. degrees in electrical engineering from Shandong University, Jinan, China, in 1989 and 1993, respectively, and the Ph.D. degree in electrical engineering from Shenyang University of Technology, Shenyang, China, in 1996. He is currently a Professor with the School of Electrical Engineering, Shandong University, Jinan, China. His research interests include permanent magnet machines, theoretical analysis and calculation of electromagnetic devices, and artificial intelligence and its application on electrical machines.

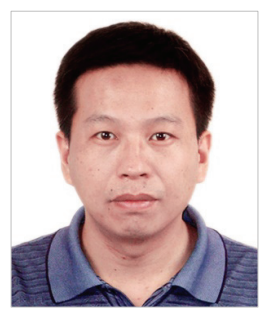

Yubo Yang He received the B.E., M.E. and $\mathrm{Ph} . \mathrm{D}$. degrees from Shandong University, Jinan, China, in 2000, 2003 and 2007 respectively all in electrical engineering. Currently he is an Assistant Professor with the School of Electrical Engineering, Shandong University, Jinan, China. His main interests include theoretical analysis and field calculation on permanent magnetic machines and special electrical machines. 